\title{
Adrenal Schwannoma: A Case Report
}

\author{
Azwa Janjua (D) \\ Muhammad Zeeshan Sarwar \\ Syed Asghar Naqi
}

Department of Surgery, East Surgical Ward, Mayo Hospital, King Edward Medical University, Lahore, Pakistan
Correspondence: Azwa Janjua Email azwamunim@gmail.com

\begin{abstract}
Schwannoma of the adrenal gland is very rare, comprising $0.2 \%$ of adrenal tumors, presenting usually as non-specific abdominal pain. We report such a rare case in a 50-year-old female. Her only complaint was right lumbar pain. Ultrasonography (USG) and computed tomography (CT) both showed a supra renal cystic mass most likely of adrenal origin. Excision of mass was performed. Post-operative course was uneventful. Histopathology showed a benign tumor of Schwann cell origin. Usually, pre-operative imaging including CT and magnetic resonance imaging (MRI) are inconclusive and diagnosis has to be confirmed on histopathology.
\end{abstract}

Keywords: adrenal tumors, schwannoma, adrenal schwannoma

\section{Introduction}

Schwannoma is a benign tumor that arises from Schwann cells of peripheral, motor, sympathetic or cranial nerves. ${ }^{1}$ Lower and upper extremities, and head and neck are the most common sites of its origin. It may also be found in areas like juxta-adrenal or retroperitoneum but visceral organs especially adrenals are a rare location. ${ }^{2}$

Phrenic nerve, vagus nerve and sympathetic trunk innervate adrenal medulla. So, schwannoma of adrenal medulla basically originates from sheaths of these nerves. ${ }^{1,2}$ These are found more in females than in males and usually in the second or fifth decade of their lives. ${ }^{2}$ We report a case of a 50 -year-old female with adrenal schwannoma who presented with right lumbar pain and fullness.

\section{Case Presentation}

A 50-yearsold woman, normotensive and normoglycemic, presented in outdoor department of Mayo Hospital Lahore in October 2018 with the complain of right lumbar pain for past 3 months. She had history of total thyroidectomy and appendectomy a few years previously with unremarkable family and medical history. A general physical examination was unremarkable with blood pressure of 130/85 $\mathrm{mmHg}$; pulse rate 72 beats/min and sinus rhythm. Blood picture and biochemical profile were in normal range. On abdominal examination, there was right lumbar fullness and minimal tenderness. The rest of the examination was un-remarkable.

Ultrasonography (USG) showed a complex cystic mass measuring $13 \times 10 \mathrm{~cm}$ in right supra renal area, not separable from upper pole of kidney, compressing and displacing IVC anteriorly and compressing the right lobe of liver as well. Mass did not show any significant flow on Doppler. On contrast-enhanced abdominal computed tomography (CT), a well circumscribed, multiloculated cystic lesion measuring $13.8 \times$ $13.1 \times 12.3 \mathrm{~cm}(\mathrm{CC} / \mathrm{AP} / \mathrm{T}$ dimensions $)$ in the right upper abdomen in supra-renal location, demonstrating internal enhancing septae and peripheral solid component. No 
internal fat or calcification was seen. Mass was abutting the right kidney, however, separable from the liver and pancreas. The right adrenal gland was not separately visualized. The lesion was displacing the liver anteriorly, right kidney inferiorly, pancreas, adjacent bowel and vessels medially. CT findings of left kidney, adrenal gland, liver, gall bladder, pancreas, spleen and bowel loops were normal. Metabolic workup was normal which included serum profile, serum metanephrine, vanillylmandelic acid (VMA) and serum cortisol levels. Diagnosis of right sided adrenal mass was made and planned for exploration. After informed consent, with an open approach, midline exploratory laparotomy was performed. On exploration, a well-encapsulated, solid-cystic mass was seen, displacing the right kidney inferiorly. The mass was completely separable from liver and was displacing the IVC anteriorly (Figure 1A and $\underline{B}$ ).

Due to the benign characteristics of the mass a partial adrenalectomy was done. Although a minimally invasive approach is a standard procedure for tumors less than $6 \mathrm{~cm}$, an open approach was preferred due large size and lack of expertise. Histopathology revealed a $14.2 \times 13 \times$ $12 \mathrm{~cm}$ circumscribed neoplastic lesion composed of hyper-

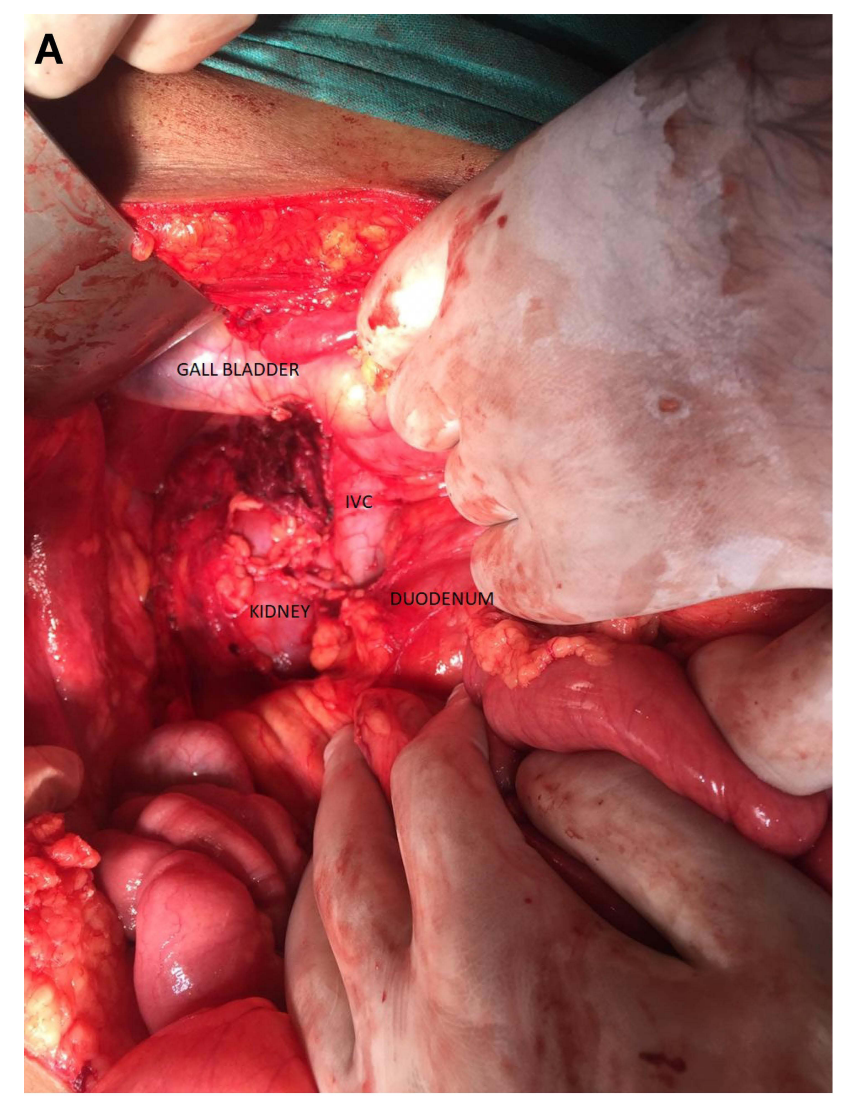

and hypo-cellular areas. In hypercellular areas the nuclei were palisading around fibrillary process (verocay body formation). In hypocellular areas, the cells were loosely textured and contained scant cytoplasm with serpentine nuclei. Thick walled hyalinized vessels were seen without significant mitotic activity. S100 and SOX-10 were positive, concluding the specimen to be benign SCHWANNOMA. Patient did well after the surgery. There were no complications and she was sent home after 72 hours. There was no recurrence of disease till 12 months post-surgery.

\section{Discussion}

Schwannoma is a nerve cell tumor that can arise almost everywhere in organs and nerve trunks except cranial nerves I and II which do not have Schwann cell sheath. ${ }^{1}$ Verocay was the first to describe them in 1908 and Antonini (1920) subclassified these tumors as the ones with hyalinization and interstitial fibrosis. ${ }^{3}$ Long standing tumors also show degenerative changes in the form of either cystic changes, fibrosis, calcification or hyalinization and have a better outcome. ${ }^{4}$ Schwannomas can be malignant as well which often are multiple and associated

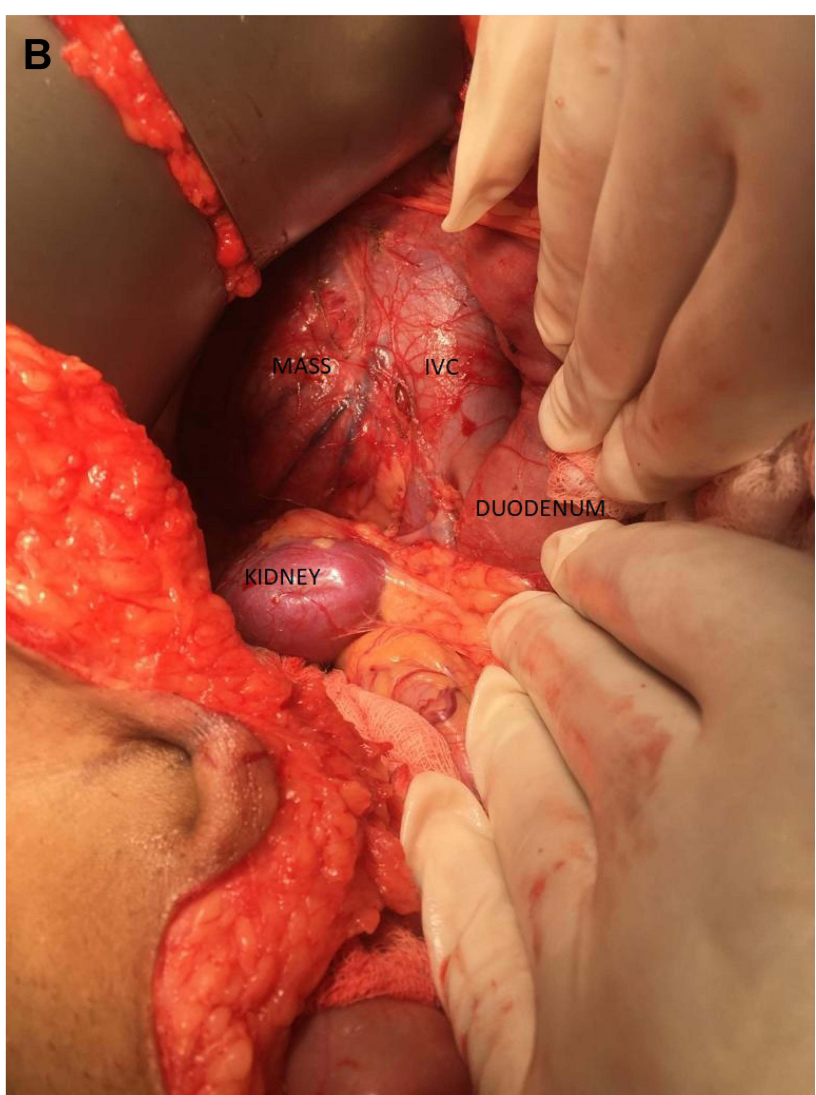

Figure I (A, B) Per operative pictures showing tumor with adjacent structures. 
with von-Recklinghausen's disease in upto $18 \%$ of the cases. ${ }^{5}$ Adrenal schwannomas originate from the sheaths of various nerves innervating the gland and compressing the cortex. Due to absence of any septum around the tumor, they appear to be arising from adrenal medulla. ${ }^{6}$ Adrenal Schwannomas occur mostly in females in the second or fifth decade of life with non-specific symptoms of abdominal or lumbar pain or discomfort. ${ }^{2}$

Imaging studies are usually not helpful in differentiating a schwannoma from other adrenal tumors, however, a combination of necrosis with arterial phase mass enhancement on CT rules out Schwannoma. ${ }^{7}$ Moreover, on CT, adrenal schwannoma appears as wellcircumscribed round or oval low-density suprarenal masse with a mean attenuation value of $30.1 \mathrm{HU}$ of solid portions during unenhanced phase. ${ }^{8}$ Whereas T1 and T2 weighted images on magnetic resonance imaging (MRI) show low signal and high signal intensity respectively. ${ }^{3}$

On histopathological examination, they consist of Antoni A and B cells which consist of high cellularity component and pure myxoid component respectively and stain positively for S100. Because of these features it is sometimes difficult to distinguish them from neurofibromas. ${ }^{9}$ In such cases calretinin helps in diagnosing schwannoma. ${ }^{10}$

Adrenal masses of more than $6 \mathrm{~cm}$ in size are generally managed by excision, either open or laparoscopic approach. $^{11}$ Laparoscopic approach includes transperitoneal and retroperitoneal techniques. Among these, retroperitoneal approach is safer in terms of morbidity. ${ }^{12}$ Open access, associated to higher mortality (2-4\%) and morbidity rates (bleeding, pulmonary and cardiac issues, pulmonary thromboembolism, wound infections), is worldwide reserved only for large tumors (diameter $>$ $6 \mathrm{~cm}$ ) and primary malignancies, based on the radicality of resection, minor tumor local recurrence, and major survival. ${ }^{13}$ Management guidelines specific for adrenal schwannomas are yet to be established as these are seldomly diagnosed pre operatively. ${ }^{11}$ The diagnosis of schwannoma is based mostly on histopathology and a surgeon must have a high index of suspicion preoperatively and must rule out other possible diagnosis if they are planning on excision of the mass only.

To date, almost 40 cases of adrenal schwannomas have been reported. ${ }^{6}$ In our case we proceeded with complete excision of mass after imaging confirmed it to be a cystic lesion and blood work showed no evidence of hyperactive adrenals. Open approach was used via midline incision as mass was massive ie around $14 \mathrm{~cm}$ in its largest diameter.
Post operatively, immunohistochemical staining and histology both confirmed the mass to be schwannoma which was at least expected pre-operatively. Hence, while dealing with benign supra renal masses, schwannoma should be kept in differentials down the list. Especially when the patient has vague abdominal symptoms and there are no systemic effects either.

\section{Conclusion}

We report on an adrenal schwannoma in a 50-year-old female patient. Pre-operative diagnosis of these schwannomas is difficult. Absolute diagnosis is only possible by surgically removing the tumor, and histopathological and immunohistochemical staining of the operative specimen.

\section{Ethical Approval}

Institutional Approval from King Edward Medical University was taken to publish the case report.

\section{Ethics and Consent}

The patient provided informed consent for publication of their case details and any accompanying images.

\section{Disclosure}

The authors report no conflicts of interest for this work.

\section{References}

1. Korets R, Berkenblit R, Ghavamian R. Incidentally discovered adrenal schwannoma. JSLS. 2007;11(1):113-115.

2. Shivalingaiah $P$, Kumar P, Bajoria S. Adrenal schwannoma treated with open adrenalectomy: a case report. Indian J Surg Oncol. 2017;9 (1):83-85. doi:10.1007/s13193-017-0715-5

3. Dell'Aversano Orabona G, Ricci D, Emili I, Serpi F, Ferrara V, Vanzulli A. Adrenal schwannoma: a case report. Case Rep. 2019;6:20190044. doi:10.1259/bjrcr.20190044

4. Antinheimo J, Sankila R, Carpen O, Pukkala E, Sainio M, Jaaskelainen J. Population-based analysis of sporadic and type 2 neurofibromatosis-associated meningiomas and schwannomas. Neurology. 2000;54(1):71-76. doi:10.1212/WNL.54.1.71

5. Rattier B, Desrousseaux B, Dereux HJ, Ampe AI. J: benign retroperitoneal pelvic schwannoma. A propos of 2 cases. $J$ Chir. 1990;127:209-212.

6. Guo Y-K, Yang Z-G, Li Y, et al. Uncommon adrenal masses: CT and MRI features with histopathologic correlation. Eur J Radiol. 2007;62 (3):359-370. doi:10.1016/j.ejrad.2006.12.011

7. Shen Y, Zhong Y, Wang H, Lu ML. MR imaging features of benign retroperitoneal paragangliomas and schwannomas. BMC Neurol. 2018;18:1. doi:10.1186/s12883-017-0998-8

8. Tang W, Yu X, Zhou L, Gao H, Wang Q, Peng W. Adrenal schwannoma: CT, MR manifestations and pathological correlation. Clin Hemorheol Microcirc. 2018;68(4):401-412. doi:10.3233/CH-170316

9. Fine SW, McClain SA, Li M. Immunohistochemical staining for calretinin is useful for differentiating schwannomas from neurofibromas. Am J Clin Pathol. 2004;122(4):552-559. doi:10.1309/AGBGTBRJ4W0 $\mathrm{BC} 7 \mathrm{LN}$ 
10. Daneshmand S, Youssefzadeh D, Chamie K, et al. Benign retroperitoneal schwannoma: a case series and review of the literature. Urology. 2003;62(6):993-997. doi:10.1016/S0090-4295(03) 00792-1

11. Oberoi A, Kataria K, Prakash O, Yadav R, Goyal A. Management of giant adrenal schwannoma. Int Surg J. 2019;6(7):2605-2608. doi:10.18203/2349-2902.isj20193003
12. Arezzo A, Bullano A, Cochetti G, et al. Transperitoneal versus retroperitoneal laparoscopic adrenalectomy for adrenal tumours in adults. Cochrane Database Syst Rev. 2018. doi:10.1002/14651858. CD011668.pub2

13. Conzo G, Gambardella C, Candela G, et al. Single center experience with laparoscopic adrenalectomy on a large clinical series. BMC Surg. 2018;18(1). doi:10.1186/s12893-017-0333-8.

\section{Publish your work in this journal}

The International Medical Case Reports Journal is an international, peer-reviewed open-access journal publishing original case reports from all medical specialties. Previously unpublished medical posters are also accepted relating to any area of clinical or preclinical science. Submissions should not normally exceed 2,000 words or 4 published pages including figures, diagrams and references. The manuscript management system is completely online and includes a very quick and fair peer-review system, which is all easy to use. Visit http://www.dovepress.com/testimonials.php to read real quotes from published authors. 